\title{
A Framework for Crop Disease Detection Using Feature Fusion Method
}

\author{
Radhika Bhagwat $^{1,2, *}$, Yogesh Dandawate ${ }^{3}$ \\ ${ }^{1}$ Department of Technology, Savitribai Phule Pune University, Pune, India \\ ${ }^{2}$ Department of Information Technology, Cummins College of Engineering for Women, Pune, India \\ ${ }^{3}$ Electronics and Telecommunication Engineering, Vishwaskarma Institute of Information Technology, Pune, India \\ Received 19 March 2021; received in revised form 10 May 2021; accepted 11 May 2021 \\ DOI: https://doi.org/10.46604/ijeti.2021.7346
}

\begin{abstract}
Crop disease detection methods vary from traditional machine learning, which uses Hand-Crafted Features (HCF) to the current deep learning techniques that utilize deep features. In this study, a hybrid framework is designed for crop disease detection using feature fusion. Convolutional Neural Network (CNN) is used for high level features that are fused with HCF. Cepstral coefficients of RGB images are presented as one of the features along with the other popular HCF. The proposed hybrid model is tested on the whole leaf images and also on the image patches which have individual lesions. The experimental results give an enhanced performance with a classification accuracy of $99.93 \%$ for the whole leaf images and $99.74 \%$ for the images with individual lesions. The proposed model also shows a significant improvement in comparison to the state-of-art techniques. The improved results show the prominence of feature fusion and establish cepstral coefficients as a pertinent feature for crop disease detection.
\end{abstract}

Keywords: crop disease detection, feature fusion, convolutional neural network, hand-crafted features, cepstral coefficients

\section{Introduction}

Agriculture is the main source of living for a major portion of India's population and contribute significantly to the country's GDP. However, the balance between the demand and supply of food due to the ever increasing population is a worrying issue. One of the reasons for this imbalance is crop loss due to pests and diseases that cause significant yield loss. Secondly, the impacts of climate changes make the conditions more alarming for the rapid spread of diseases. These diseases, if detected in the early stages, can help maintain the yield and control the economic loss. The traditional way of detecting diseases by a plant pathologist is time consuming and costly. The other traditional method is using pesticides. Normally, however, pesticides are used randomly on the entire canopy, and if they are used in excess, soil pollution can be caused and the soil quality can be degraded. Since the past few decades, crop disease detection using image processing techniques has been proved to be fast and accurate to overcome the constraints of the conventional methods.

All the earlier work done in this field focused on the traditional machine learning algorithms using Hand-Crafted Features (HCF) that required domain knowledge to reduce complexities in the input data. However, a much bounded aspect of the problem is covered by these features and their capability degrades on substantial datasets. Recently, the advent of deep learning technology has resulted in remarkable improvement in a broad range of computer vision problems. In the agriculture domain, the significance of deep learning has been proved [1]. Deep learning techniques hierarchically learn sophisticated features from the input data by the convolutional kernel as the network goes deeper. If these deep features having high representative abilities are fused with HCF, more extensive information related to the data can be captured. Recently, researchers in many domains

* Corresponding author. E-mail address: radhika.bhagwat@cumminscollege.in, radbhag@gmail.com 
[2-3] have started exploring the potential of fusion at different levels to increase classification accuracy. Just as in numerous other domains, e.g., in the plant pathology sector, researchers have attempted to improve the classification performance by various fusion techniques.

Cruz et al. [4] presented an algorithm for detection of olive quick decline disorder by data fusion with deep learning. The authors fused relevant $\mathrm{HCF}$ at various levels of abstraction to enhance the detection performance. The study fused the order statistics, texture, shape features, and geometric relationships at the fully connected layers of a modified LeNet and achieved an improved accuracy of $98.60 \%$. Çuğu et al. [5] fused deep features and HCF for leaf based plant classification. They combined 56 features (shape, contour, color, and texture) obtained by using several image processing methods and deep features (4096) obtained by using fine tuning of Caffe framework to train a Linear Support Vector Machine (LSVM) for classification of 57 tree species. The work fused HCF with the features obtained from the first fully connected (fc6) and the second (fc7) fully connected layer, and the comparison of these results found that the fusion with the fc6 layer gave the highest accuracy of $90.5 \%$ on the Treelogy dataset and $99.68 \%$ on the Flavia dataset.

Zhang et al. [6] proposed a model with multi-scale convolution (inception) and feature fusion (concat) layer for cucumber leaf disease detection. The inception layer extracted multi-scale features while the concat layer fused those features to get a comprehensive feature map that helped the model learn complete features of the input data giving an accuracy of $94.65 \%$. Jiang et al. [7] used an inception module to extract multi-scale features of apple leaf diseases and the feature fusion was achieved by rainbow concatenation giving an improved detection performance of $78.80 \%$ maP. Genshang et al. [8] fused the traditional machine learning and deep learning methods for disease detection of tea leaves. They extracted color features and texture features and segmented the lesion using a support vector machine while they used the deep learning VGG16 model to recognize the disease resulting in an average classification accuracy of 90\%. Hu et al. [9] used multi-feature fusion for rape plant disease recognition. By using color and texture features and utilizing the Dempster-Shafer rule of evidence, feature fusion was achieved that gave a recognition rate of $97.09 \%$. Bansal and Kumar [10] proposed plant disease recognition using post processing fusion. The work combined the prediction output of multiple deep learning algorithms and assigned the maximum score as the final class output. Liu and Wang [11] fused features of different levels of the image pyramid of Yolo v3 model to get feature maps of different scales for tomato disease and pest detection.

While all the work mentioned in the literature related to feature fusion techniques make use of the traditional spatial features like texture, color, shape, geometric properties, etc., recent research is being focused on analyzing how features extracted in other domains can be used with deep architectures. Cepstral analysis is the most widely used technique in speech processing applications. However, the use of 2D cepstrum analysis has been studied for feature extraction in 2D images as well.

Cakır [12] used 2D cepstrum based technique for extracting features for face recognition. Gupta et al. [13] used Mel Frequency Cepstral Coefficients (MFCC) for hand gesture recognition. The work converted hand gesture images into 1D signal for extracting cepstral features and used support vector machine for classification. Hashad et al. [14] used cepstral features for fingerprint recognition, while Barpanda et al. [15] used wavelet cepstrum features for iris recognition. They extracted wavelet MFCC from the images. Their experiments gave superior results when compared to the state-of-art techniques. Awad et al. [16] used MFCC for resolution enhancement in images for pattern recognition applications. Their work converted the image into 1D signal which was used for extracting features. 2D cepstrum is an extension of 1D frequency cepstrum and is defined as the Discrete Cosine Transform (DCT) or Inverse Discrete Fourier Transform (IDFT) of logarithm of power spectrum of the image. $2 \mathrm{D}$ cepstrum is computationally effective and involves logarithmic operation that makes it amplitude invariant which also leads to illumination invariance. As it is based on frequency domain, it is shift and scale invariant in which the high frequency components give the fine details while the low frequency components represent the large scale information in the image. 
Motivated by the work done in literature, in this study, a hybrid model for crop disease detection based upon the fusion of $\mathrm{HCF}$ and deep features is presented. The work done in literature for feature fusion focuses on the fusion of standard HCF with deep features. However, the proposed model suggests the use of cepstral coefficients as one of the HCF along with the other standard texture and shape features. The deep features are extracted from a Convolutional Neural Network (CNN) [17] while the use of cepstral coefficients is done as one of the HCF. Experimental analysis is done using two datasets, one with whole leaf images and the other with image patches of lone lesions and spots. The experimental results show remarkable advancements with the fusion framework and prove cepstral coefficients as a viable feature for image classification in crop disease recognition. The results also demonstrate the significance of the features extracted in the frequency domain in deep architectures. Comparative experiments with the state-of-art techniques also show substantial improvement with the fusion of both types of features.

The major contributions of the study are: 1) A hybrid framework is proposed to fuse deep features with the extracted HCF to enhance the classification performance. 2) The potential of cepstral coefficients as one of the HCF for crop disease detection using RGB images is studied. The arrangement of the study is as follows: Material and methods are described in section 2. Results and discussion are presented in Section 3 followed by the conclusion in section 4 .

\section{Materials and Methods}

This section gives details of the database used for the work and explains the HCF and deep features utilized for the classification of crop diseases.

\subsection{Dataset}

The work uses the Plant Village database [18] and the Digipathos database (database for plant disease symptoms (PDDB)) [19-21] for training and testing the hybrid model. The Plant Village dataset (dataset 1) has healthy and diseased whole leaf images for 38 categories with 54,308 total images. The Digipathos dataset (dataset 2) has image patches of lone lesions and spots of 53 categories and 43,106 total images. The use of the whole leaf images and the image patches having individual lesions and spots is done to assess the efficiency of the proposed model in capturing global as well as local patterns. Dataset 1 has an image size of $256 \times 256$ while images in dataset 2 are of varied sizes. All the images are preprocessed to make their sizes compatible according to the model architecture. Fig. 1 shows the sample images of the two datasets.

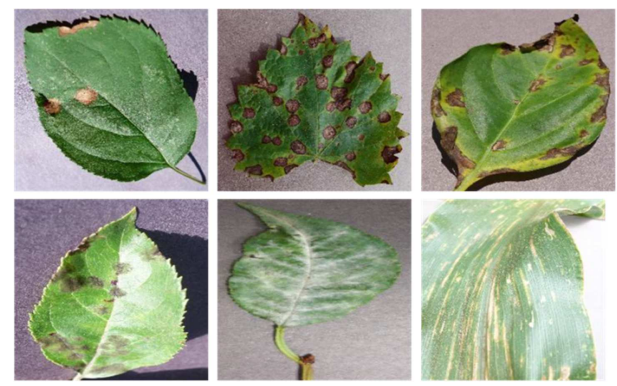

(a) Plant Village dataset (dataset 1)

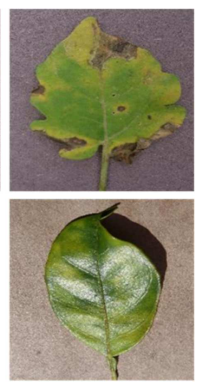

Fig. 1 Sample images

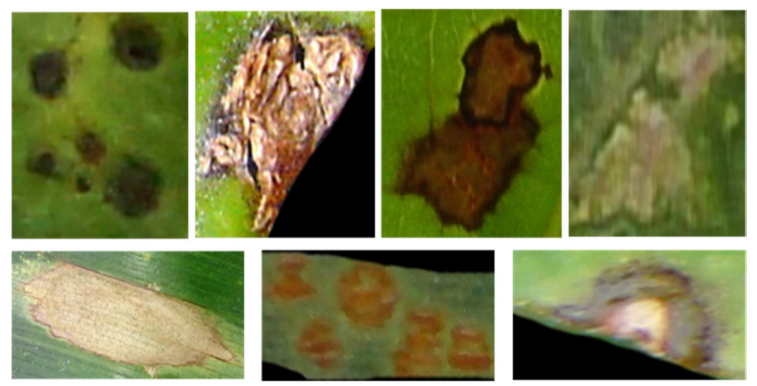

(b) Digipathos dataset (dataset 2)

\subsection{Hand-crafted features (HCF)}

Two groups of conventional HCF of texture and shape are used in the work. Gray Level Co-occurrence Matrix (GLCM) and Gabor filters are applied for extraction of texture features while for extracting shape features Hu moments and region properties are used. Along with these standard features, the work proposes the use of cepstral coefficients as one of the effective HCF. 


\subsubsection{Standard texture and shape features}

The study of texture in an image helps get knowledge about the spatial alignment of color or intensities while shape features are visual features that helps explain the object. Several techniques are used in literature for extracting texture features. In the present work, texture features are obtained using GLCM and Gabor filters. Using GLCM, four statistical features,namely contrast, correlation, energy, and homogeneity at four orientations $0^{\circ}, 45^{\circ}, 90^{\circ}$, and $135^{\circ}$ are used to get 16 features. Gabor filters, another popular technique used for extracting texture, have prominent responses at edges and also at points of texture changes. Five scales and eight orientations are used in the work to get 202 features. Hu moments and region properties like area, convex hull, perimeter, etc. are used as shape features. The seven Hu moments are the shape descriptors that give an advantage of invariance to translation, scale, and rotation.

\subsubsection{The proposed cepstrum analysis}

Cepstrum analysis is one of the standard and widely-used techniques used for speech processing applications. However, studies have also used cepstral analysis for 2D images and its implementations, e.g., face recognition [12], hand gesture recognition [13], fingerprint recognition [14], iris feature extraction [15], resolution enhancement [16], etc.

Influenced by the achievements of cepstrum analysis in several areas, its exploitation as one of the possible HCF to access its effect on crop disease detection is proposed. 2D cepstrum is defined as the DCT or IDFT of logarithmic power spectrum of the image. It is a spectrum of log spectrum that is similar to the inverted frequency and hence is called the quefrency domain. $2 \mathrm{D}$ cepstrum is a computationally efficient technique and involves using Fourier transform and logarithm operation. It gives the structural information where the high frequency components show the fine details while low frequency components give the large scale information. Most of the natural images are low pass by their nature and their energy drops at high frequency, because of which the higher values of low frequency components subdue the impact of high frequency components. In the proposed cepstrum analysis, the Discrete Fourier Transform (DFT) domain data is normalized to overcome the dominance of low frequency components, and is divided into blocks to compute the power of each block. The Fourier transform involved in the process makes the features invariant to translation while the logarithm operation makes them invariant to amplitude changes (scale invariance). The invariance to amplitude changes also leads to illumination invariance [12].

Although all the work in literature on 2D cepstrum is done on gray images, in this work, the use of RGB images for extracting cepstral coefficients to assess the correlation between the color components for crop disease detection is proposed. Fig. 2 shows the procedure for cepstral coefficient extraction, and the algorithm below explains the steps for feature extraction using the proposed cepstral analysis process. All the images used for the work are of size $128 \times 128$. As far as is known, the proposed technique is the first effort in existing literature to exploit cepstrum analysis on RGB images.

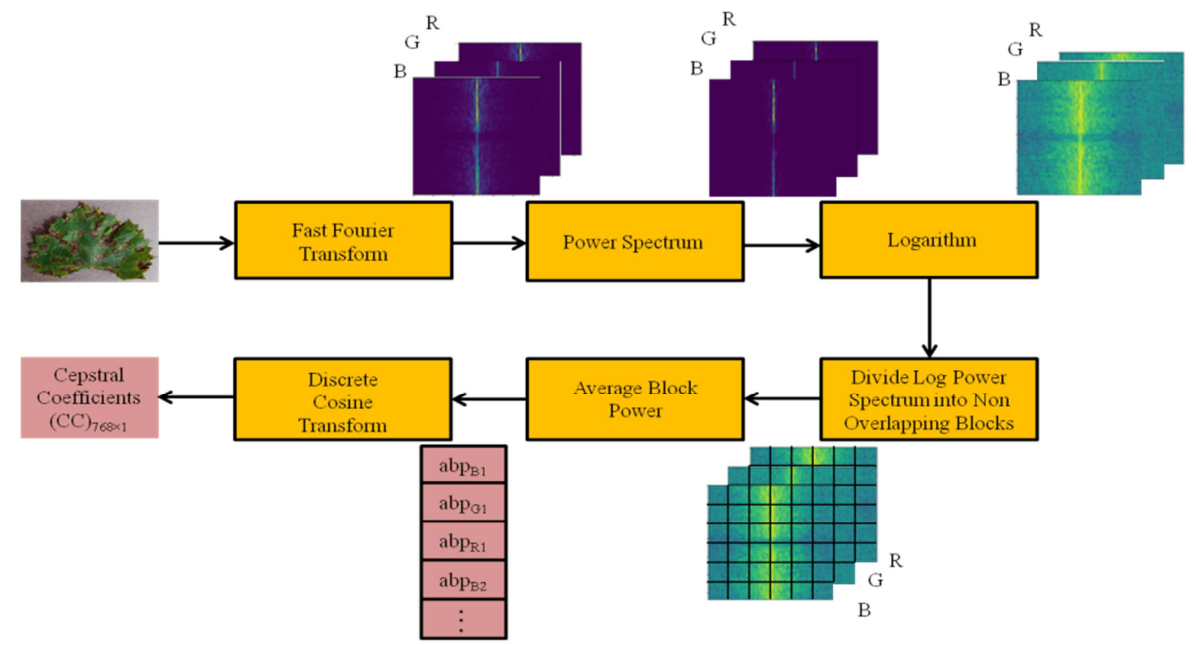

Fig. 2 Process of cepstral coefficient extraction 


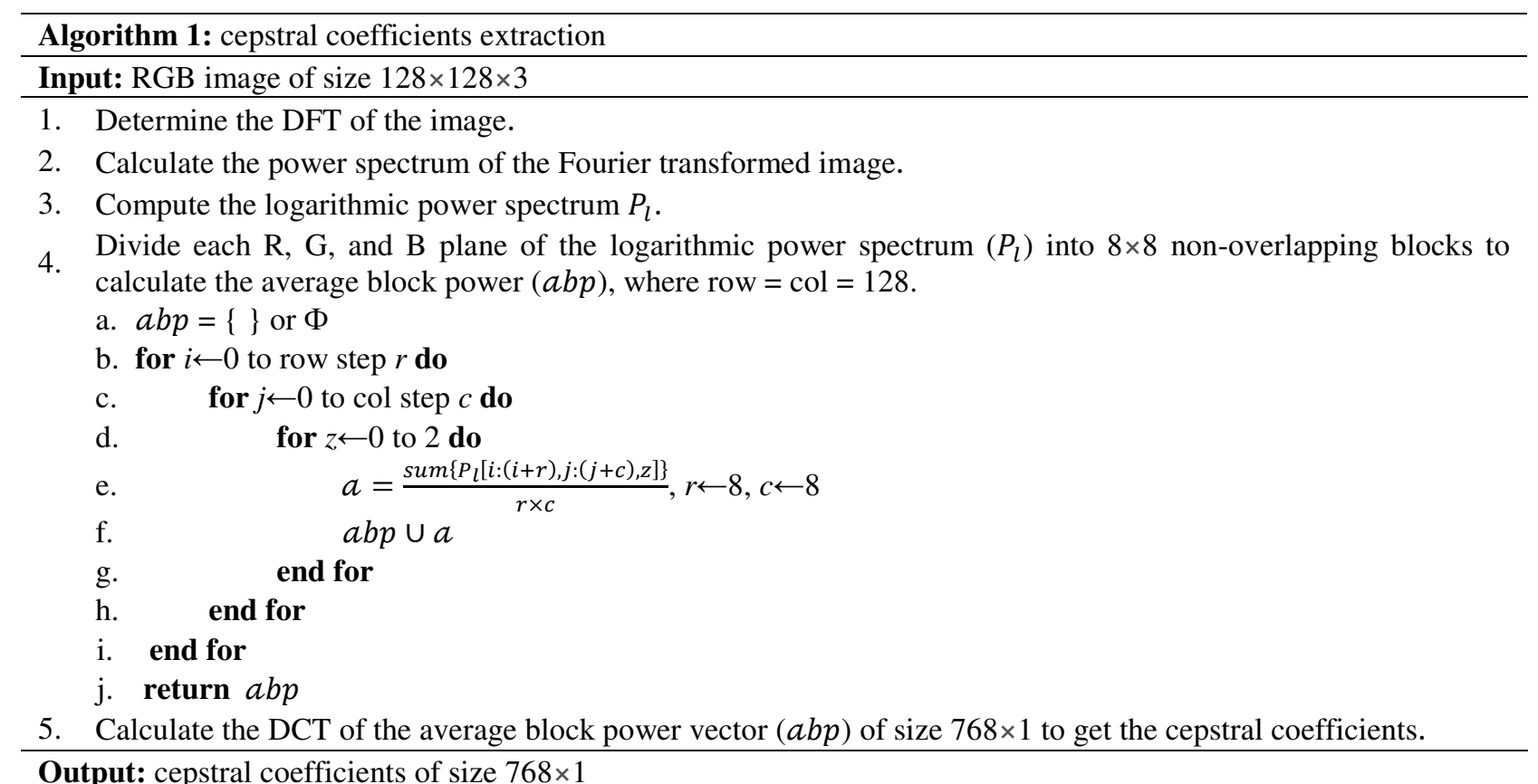

\subsection{Deep features}

CNN is amongst the major deep learning methods. It typically includes convolutional layers, pooling layers, and fully connected layers. Using suitable filters, the convolutional layers capture the most significant features that represent the input data. The initial convolutional layers learn the lower level features while more prominent features related to the data are extracted at higher layers. Rectified Linear Unit (ReLu) activation, filter size of $3 \times 3$, and default stride rate of 1 are used for the convolutional layers in the presented work. The filter numbers are 32, 64, 128, and 192 for the four convolutional layers. The pooling layer reduces the size of the extracted features for dimensionality reduction. To reduce the required computational power, Max pooling is used with a filter dimension of $2 \times 2$. Depending upon the input data, the number of convolutional and pooling layers can be increased to capture details even more. However, this increases the cost of computations. Fig. 3 shows the technique of deep feature extraction. The achieved feature map is of the size $4800 \times 1$.

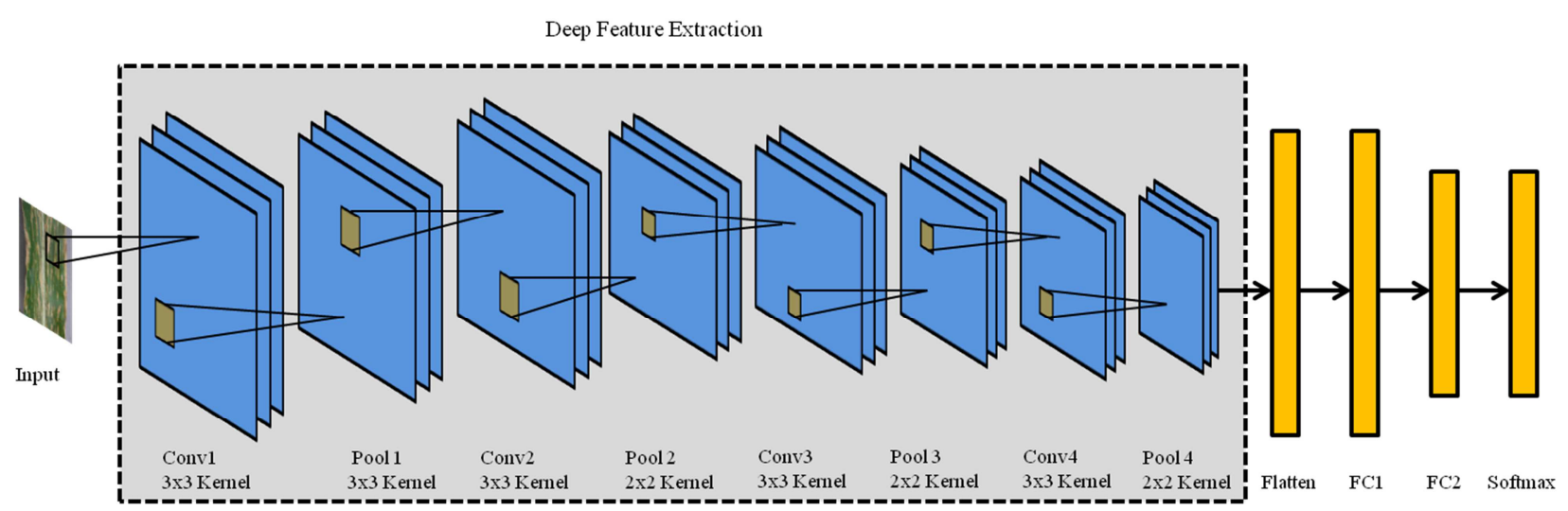

Fig. 3 Deep feature extraction

\subsection{The proposed fusion procedure}

The framework for the fusion of HCF and deep features for crop disease detection is shown in Fig. 4. Initially, the images are resized to the size of $128 \times 128$. As can be seen from Fig. 4, there are three main phases in the proposed work. In the first phase, the images are used for extracting the standard handcrafted texture and shape features and the suggested cepstral coefficients. In the second phase, deep features are extracted using the CNN architecture as shown in Fig. 3. In the third phase, both types of features are flattened and fused into a feature vector. Fully connected layers with a softmax classifier are used to 
categorize the crop diseases. The use of batch normalization is done with each convolutional layer and fully connected layer. A drop-out with 0.5 probability is used after the fully connected layers to avoid overfitting. The loss function used is cross entropy and the adaptive moment estimation optimizer is used with a learning rate of 0.0001 . The batch size is fixed at 32 .

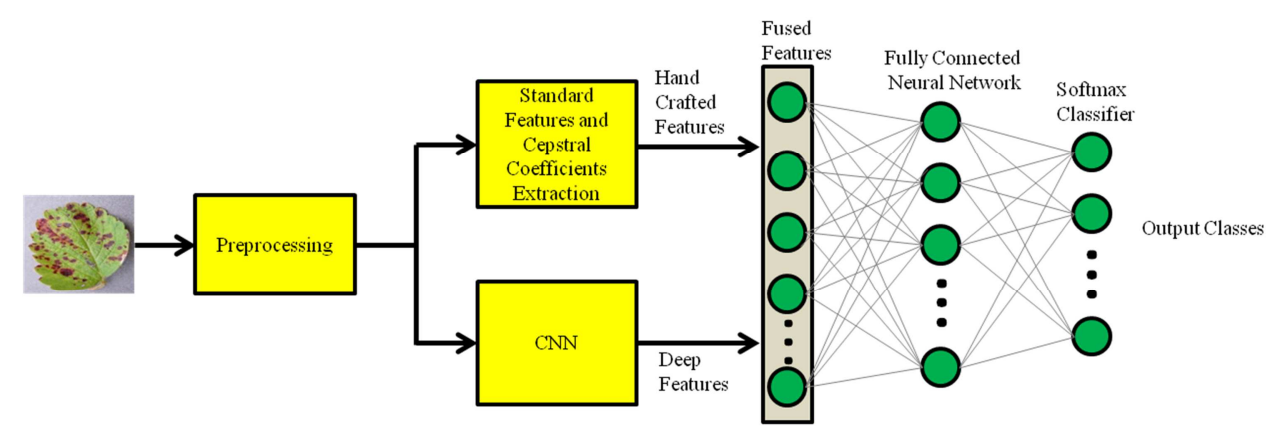

Fig. 4 Framework for feature fusion

\section{Results and Discussion}

This section presents the classification results for HCF, deep learning features, and the fusion of both features. The results are also checked with the existing advanced CNN architectures. The classification performance is assessed using accuracy, positive predictive value (precision), sensitivity (recall), F-score, and the area under the Receiver Operating Characteristic (ROC) curve. As classes in both datasets are of different sizes, the Matthews Correlation Coefficient (MCC) is also utilized to evaluate the classifier performance.

\subsection{Cepstarl coefficients compared with standard HCF}

In correspondence with cepstral coefficients, there are two variable parameters namely, block size and the overlap between adjoining blocks. The work is tested for varied block sizes and overlapping percentages, and it is found that a block size of $8 \times 8$ and non-overlapping blocks give the best results. Therefore, this combination is used to get the optimal results. The classification performance of cepstral coefficients is compared with the popular HCF using the traditional machine learning algorithm. The experimental outcomes for dataset 1 are shown in Table 1. As reflected in the table, the suggested cepstrum analysis gives the best efficiency compared to the standard HCF.

Table 1 Comparison of cepstral coefficients with conventional HCF using traditional machine learning algorithm

\begin{tabular}{|c|c|c|c|c|}
\hline Ref. & Crop & Feature type & Classifier & Result \\
\hline$[22]$ & Citrus & $\begin{array}{c}\text { Color, texture, and } \\
\text { geometric features }\end{array}$ & Average accuracy $=92.435 \%$ \\
\hline$[23]$ & Multiple crops & $\begin{array}{c}\text { Texture features using color } \\
\text { co-occurence matrix }\end{array}$ & $\begin{array}{c}\text { SVM with the proposed GA } \\
\text { method }\end{array}$ & Accuracy $=95.71 \%$ \\
\hline$[24]$ & Tomato & Textural patterns & SVM & Accuracy $=91.5 \%$ \\
\hline$[25]$ & Multiple crops & LBP histogram & $\begin{array}{c}\text { One-class SVM with the nearest } \\
\text { support vector }\end{array}$ & Accuracy $=95 \%$ \\
\hline$[26]$ & Not specified & Region growing algorithm & $\begin{array}{c}\text { Bacterial Foraging Optimization } \\
\text { Based Radial Basis Function } \\
\text { Neural Network (BRBFNN) }\end{array}$ & $\begin{array}{c}\text { Accuracy (validation evaluation } \\
\text { partition coefficient) }=0.8621\end{array}$ \\
\hline$[27]$ & Tomato & Texture and color & SVM & Accuracy $=85.1 \%$ \\
\hline $\begin{array}{c}\text { This } \\
\text { study }\end{array}$ & Multiple crops & $\begin{array}{c}\text { Texture features using } \\
\text { GLCM (HCF1) }\end{array}$ & Accuracy $=96.1 \%$ \\
\hline $\begin{array}{c}\text { This } \\
\text { study }\end{array}$ & Multiple crops & $\begin{array}{c}\text { Texture features using } \\
\text { Gabor filters (HCF2) }\end{array}$ & ANN & Accuracy $=94.4 \%$ \\
\hline $\begin{array}{c}\text { This } \\
\text { study }\end{array}$ & Multiple crops & Hu moments (HCF3) \\
\hline $\begin{array}{c}\text { This } \\
\text { study }\end{array}$ & Multiple crops & $\begin{array}{c}\text { Region (geometric) } \\
\text { properties (HCF4) }\end{array}$ & ANN \\
\hline $\begin{array}{c}\text { This } \\
\text { study }\end{array}$ & Multiple crops & $\begin{array}{c}\text { Cepstral coefficients } \\
\text { (HCF5) }\end{array}$ & ANN & Accuracy $=95.1 \%$ \\
\hline
\end{tabular}




\subsection{Performance using deep features}

A CNN model [17] as shown in Fig. 3 is used to check the performance of the deep features. The performance comparison of the model shown in Fig. 3 with other CNN architectures mentioned in literature is shown in Table 2. As indicated in the table, the deep model gives the best performance for the whole leaf images as well as for the images of individual lesions and spots, indicating that it can successfully capture the global as well as local pattern in the image and use them for classification. A comparison of Table 2 with Table 1 indicates the superiority of deep features over HCF.

Table 2 Comparison of CNN model with other deep architectures

\begin{tabular}{|c|c|c|c|c|}
\hline Ref. & Crop & Dataset & Model/architecture & Result \\
\hline$[7]$ & Apple & Whole leaf & GoogLeNet (inception module) & mAP $=78.80 \%$ \\
\hline$[21]$ & 14 crops & Individual lesion and spots & GoogLeNet & Accuracy $>75 \%$ \\
\hline$[28]$ & 13 crops & Whole leaf & CNN & Accuracy $=96.46 \%$ \\
\hline$[29]$ & $\begin{array}{c}\text { Pearl } \\
\text { millet }\end{array}$ & Millet crop & CNN (VGG16) & $\begin{array}{c}\text { Accuracy }=95 \%, \text { Precision }=90.50 \% \\
\text { Recall }=94.50 \%, \text { F1-score }=91.75 \%\end{array}$ \\
\hline$[30]$ & Banana & Various parts of plants & $\begin{array}{c}\text { CNN (ResNet50, InceptionV2, } \\
\text { and MobileNetV1) }\end{array}$ & Accuracy $=70 \%-99 \%$ \\
\hline$[17]$ & 14 crops & Whole leaf & CNN & Accuracy $=99.85 \%$ \\
\hline$[17]$ & 16 crops & Individual lesion and spots & CNN & Accuracy $=99.6 \%$ \\
\hline
\end{tabular}

\subsection{Fusion of deep features and HCF}

The results obtained in sections 3.1 and 3.2 are the motivation for carrying out the fusion of HCF and deep features for crop disease detection. The fusion framework is tested for various HCF used in section 3.1. The classification results for dataset 1 and dataset 2 are demonstrated in Table 3 and Table 4 respectively. As reflected in the tables, the fusion of deep features with Gabor features, Hu moments, and regional properties do not show any improvement in the classification performance when compared to deep features alone. The fusion of deep features with texture features and cepstral coefficients show the improved results for dataset 1 . The fusion of deep features $(4800 \times 1)$ with cepstral coefficients $(768 \times 1)$ gives the best efficiency for both datasets. Thus, all the further experimentations are done by using the fusion of deep features with cepstral coefficients with final feature vector size of $5568 \times 1$.

Table 3 Classification performance of feature fusion for dataset 1

\begin{tabular}{|c|c|c|c|c|c|}
\hline Features & Accuracy & Positive predictive value & Sensitivity & F-score & MCC \\
\hline Only deep features & 0.9985 & 0.97 & 0.97 & 0.97 & 0.9872 \\
\hline Fusion of deep features with $\mathrm{HCF}_{1}$ & 0.9987 & 0.97 & 0.97 & 0.97 & 0.9837 \\
\hline Fusion of deep features with $\mathrm{HCF}_{2}$ & 0.992 & 0.95 & 0.95 & 0.95 & 0.9831 \\
\hline Fusion of deep features with $\mathrm{HCF}_{3}$ & 0.9891 & 0.94 & 0.94 & 0.93 & 0.9823 \\
\hline Fusion of deep features with $\mathrm{HCF}_{4}$ & 0.9883 & 0.9 & 0.89 & 0.89 & 0.978 \\
\hline Fusion of deep features with $\mathrm{HCF}_{5}$ & 0.9993 & 0.99 & 0.99 & 0.99 & 0.9912 \\
\hline
\end{tabular}

${ }^{*} H C F_{1}=$ Texture features using GLCM, HCF $=$ Texture features using Gabor filters, $H C F_{3}=H u$ moments, $\mathrm{HCF}_{4}=$ Region properties, $\mathrm{HCF}_{5}=$ Cepstral coefficients

Table 4 Classification performance of feature fusion for dataset 2

\begin{tabular}{|c|c|c|c|c|c|}
\hline Features & Accuracy & Positive predictive value & Sensitivity & F-score & MCC \\
\hline Only deep features & 0.996 & 0.94 & 0.93 & 0.93 & 0.9829 \\
\hline Fusion of deep features with $\mathrm{HCF}_{1}$ & 0.9923 & 0.93 & 0.93 & 0.93 & 0.9811 \\
\hline Fusion of deep features with $\mathrm{HCF}_{2}$ & 0.9911 & 0.92 & 0.91 & 0.91 & 0.978 \\
\hline Fusion of deep features with $\mathrm{HCF}_{3}$ & 0.986 & 0.89 & 0.89 & 0.88 & 0.964 \\
\hline Fusion of deep features with $\mathrm{HCF}_{4}$ & 0.986 & 0.88 & 0.87 & 0.87 & 0.961 \\
\hline Fusion of deep features with $\mathrm{HCF}_{5}$ & 0.9974 & 0.95 & 0.94 & 0.94 & 0.9852 \\
\hline
\end{tabular}

${ }^{*} H C F_{1}=$ Texture features using GLCM, HCF $=$ Texture features using Gabor filters, $H C F_{3}=H u$ moments, $\mathrm{HCF}_{4}=$ Region properties, $\mathrm{HCF}_{5}=$ Cepstral coefficients 
The convolutional layers in the deep learning models capture the spatial information of images while the cepstral analysis captures the discriminative frequency features. The combination of the information obtained from both domains gives a more comprehensive representation of the input data thus improving the evaluation results. It also illustrates how frequency domain features are useful with deep architectures. The performance of the model for the whole leaf images is higher than that obtained for the individual lesions, as the model extracts different levels of information in both cases. In the case of whole leaf images, the model captures global features that describe the comprehensive structure while in the case of individual lesions and spots the model tends to capture only local details. In both cases, the proposed work gives an improved performance.

Figs. 5 and 6 present the ROC curves for both datasets with and without fusion (only deep features). The ROC curve for each output class is represented by a different color as indicated in Tables 5 and 6. Fig. 5 (a) and Fig. 6 (a) show the Area Under the Curve (AUC) for various output classes using only deep features. Fig. 5(b) and Fig. 6(b) show the AUC for various output classes using feature fusion. As can be seen from the figures, the AUC for various output classes using feature fusion is superior as compared to the AUC using only deep features indicating improved distinguishing capability of the proposed model with feature fusion. Tables 5 and 6 show the performance evaluation for dataset 1 and dataset 2 respectively. As the datasets are imbalanced, the weighted average is considered for performance metrics. Dataset 2 with images of individual lesions and spots attains a weighted precision average of 0.95 while dataset 1 with whole leaf images attains 0.99 . Lower precision and accuracy for images with individual lesions and spots is evident due to two reasons: only local information is captured, and multiple plant species can have similar disease symptoms.

A comparison of the proposed hybrid model with the state-of-art CNN methods mentioned in the literature is shown in Fig. 7. As indicated in the figure, the proposed fusion model (the fusion of cepstral coefficients with deep features) gives the best result.

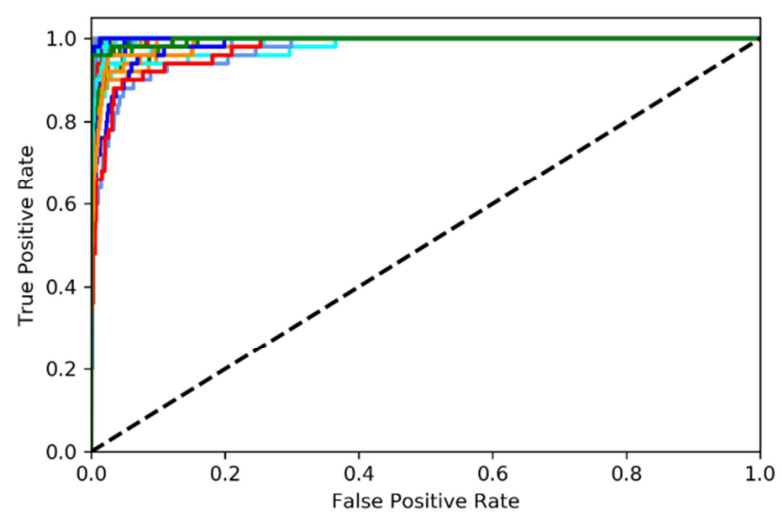

(a) Only deep features

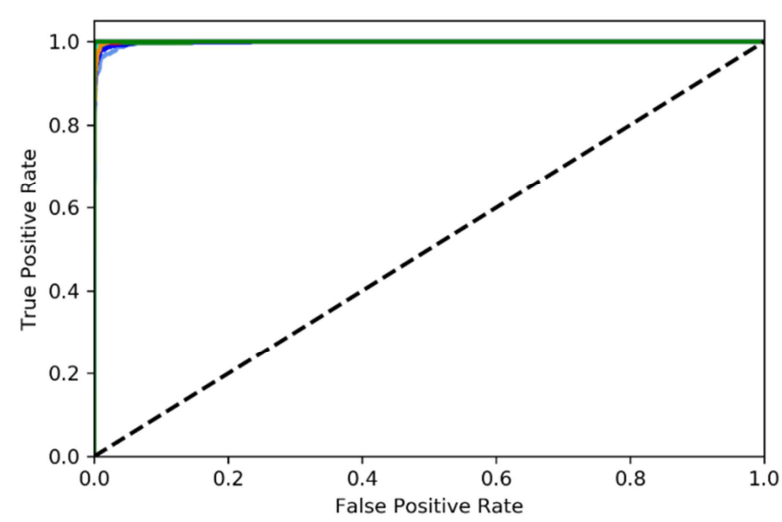

(b) Fusion of deep features and cepstral coefficients

Fig. 5 ROC for dataset 1 (output classes are indicated by various line colors)

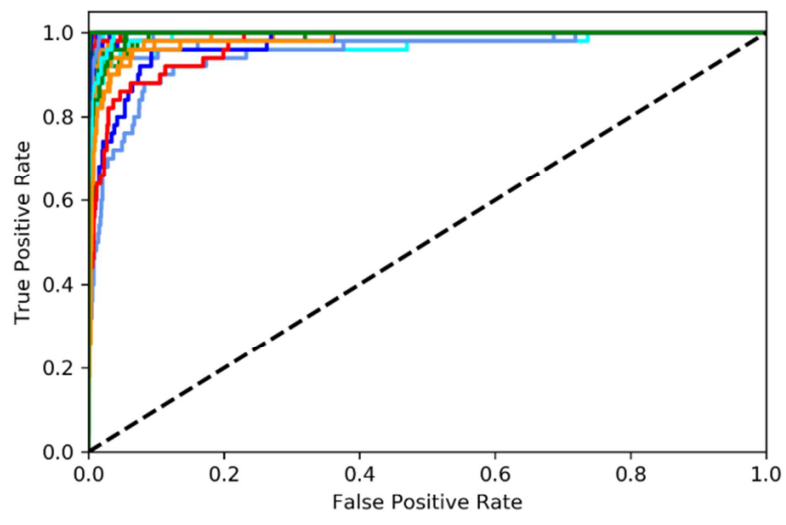

(a) Only deep features

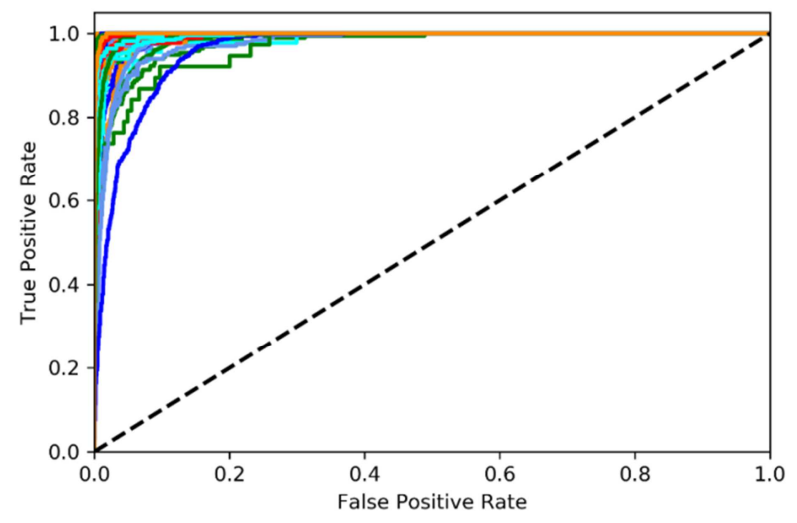

(b) Fusion of deep features and cepstral coefficients

Fig. 6 ROC for dataset 2 (output classes are indicated by various line colors) 
Table 5 Performance evaluation for dataset 1 (using Plant Village database for the whole leaf images)

\begin{tabular}{|c|c|c|c|c|c|c|}
\hline Group & Group name & Positive predictive value & Sensitivity & F-Score & ROC_AUC & $\begin{array}{c}\text { ROC_AUC } \\
\text { line color }\end{array}$ \\
\hline W0 & Scab in apple & 0.96 & 1 & 0.98 & 0.99 & \\
\hline $\mathrm{W} 1$ & Cedar apple rust in apple & 0.97 & 0.94 & 0.95 & 0.99 & 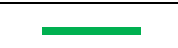 \\
\hline $\mathrm{W} 2$ & Healthy apple & 1 & 0.96 & 0.98 & 0.99 & \\
\hline W3 & Frogeye spot in apple & 1 & 0.98 & 0.99 & 0.99 & 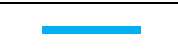 \\
\hline $\mathrm{W} 4$ & Healthy blueberry & 0.99 & 1 & 0.99 & 0.99 & 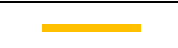 \\
\hline W5 & Healthy cherry & 1 & 1 & 1 & 1 & 2 \\
\hline W6 & Powdery mildew in cherry & 1 & 1 & 1 & 0.99 & \\
\hline W7 & Gray leaf spot in corn & 0.92 & 0.76 & 0.84 & 0.99 & \\
\hline W8 & Common rust in corn & 1 & 0.99 & 1 & 0.99 & \\
\hline W9 & Healthy corn & 0.89 & 0.97 & 0.92 & 0.99 & \\
\hline W10 & Northern leaf blight in corn & 0.99 & 1 & 0.99 & 0.99 & \\
\hline W11 & Black rot in grape & 1 & 0.99 & 0.99 & 0.99 & \\
\hline W12 & Esca black measles in grape & 1 & 1 & 1 & 0.99 & \\
\hline W13 & Healthy grape & 1 & 1 & 1 & 0.99 & \\
\hline W14 & Leaf blight in grape & 1 & 1 & 1 & 1 & - \\
\hline W15 & Citrus greening in orange & 1 & 1 & 1 & 1 & \\
\hline W16 & Bacterial spot in peach & 1 & 1 & 1 & 0.99 & \\
\hline W17 & Healthy peach & 1 & 1 & 1 & 1 & \\
\hline W18 & Bacterial spot in pepper bell & 1 & 0.97 & 0.98 & 0.99 & \\
\hline W19 & Healthy pepper bell & 1 & 1 & 1 & 0.99 & 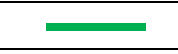 \\
\hline W20 & Early blight in potato & 0.98 & 1 & 0.99 & 0.99 & - \\
\hline W21 & Healthy potato & 0.97 & 0.96 & 0.97 & 0.99 & \\
\hline W22 & Late blight in potato & 0.91 & 0.93 & 0.92 & 0.99 & \\
\hline W23 & Healthy raspberry & 0.95 & 0.99 & 0.97 & 0.99 & \\
\hline W24 & Healthy soybean & 0.99 & 1 & 1 & 0.99 & \\
\hline W25 & Powdery mildew in squash & 1 & 1 & 1 & 0.99 & \\
\hline W26 & Healthy strawberry & 0.99 & 0.99 & 0.99 & 0.99 & \\
\hline W27 & Leaf scorch in strawberry & 0.99 & 1 & 0.99 & 1 & \\
\hline W28 & Bacterial spot in tomato & 1 & 0.98 & 0.99 & 0.99 & \\
\hline W29 & Early blight in tomato & 0.93 & 0.94 & 0.94 & 0.99 & \\
\hline W30 & Healthy tomato & 0.97 & 0.94 & 0.96 & 0.99 & \\
\hline W31 & Late blight in tomato & 0.97 & 0.99 & 0.98 & 0.99 & \\
\hline W32 & Leaf mold in tomato & 0.98 & 0.98 & 0.98 & 0.99 & \\
\hline W33 & Septoria leaf spot in tomato & 0.98 & 0.97 & 0.97 & 0.99 & $\longrightarrow$ \\
\hline W34 & Spider mites in tomato & 0.95 & 0.96 & 0.96 & 0.99 & $=$ \\
\hline W35 & Target spot in tomato & 1 & 1 & 1 & 0.99 & $=$ \\
\hline W36 & Mosaic virus in tomato & 0.97 & 1 & 0.99 & 0.99 & \\
\hline W37 & Yellow leaf curl virus in tomato & 0.98 & 1 & 0.99 & 0.99 & \\
\hline- & Average (weighted) & 0.99 & 0.99 & 0.99 & - & - \\
\hline
\end{tabular}

* The ROC_AUC column in the table is with reference to Fig. 5.

Table 6 Performance evaluation for dataset 2 (using Digipathos database for spots and lesions)

\begin{tabular}{|c|c|c|c|c|c|c|}
\hline Group & Group name & Positive predictive value & Recall & F-Score & ROC_AUC & $\begin{array}{c}\text { ROC_AUC } \\
\text { line color }\end{array}$ \\
\hline S0 & Alternaria leaf spot in cabbage & 1 & 0.97 & 0.97 & 0.99 & \\
\hline S1 & Algae in cashew & 0.96 & 0.92 & 0.92 & 0.98 & - \\
\hline S2 & Angular leaf spot in cashew & 0.95 & 0.94 & 0.94 & 0.99 & \\
\hline S3 & Anthracnose in cashew & 0.93 & 0.94 & 0.93 & 0.99 & \\
\hline S4 & Black mould in cashew & 0.94 & 0.88 & 0.88 & 0.99 & - \\
\hline S5 & Bacterial blight in cassava & 0.91 & 0.91 & 0.89 & 0.99 & - \\
\hline S6 & Green mite in cassava & 0.95 & 0.92 & 0.93 & 0.99 & - \\
\hline S7 & White leaf spot in cassava & 0.93 & 0.91 & 0.9 & 0.99 & - \\
\hline
\end{tabular}


Table 6 Performance evaluation for dataset 2 (using Digipathos database for spots and lesions) (continued)

\begin{tabular}{|c|c|c|c|c|c|c|}
\hline Group & Group name & Positive predictive value & Recall & F-Score & ROC_AUC & $\begin{array}{c}\text { ROC_AUC } \\
\text { line color }\end{array}$ \\
\hline S8 & Algae in citrus & 0.94 & 0.96 & 0.91 & 0.99 & \\
\hline S9 & Canker in citrus & 0.95 & 0.95 & 0.95 & 0.99 & 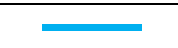 \\
\hline S10 & Greasy spot in citrus & 0.96 & 1 & 0.96 & 0.99 & 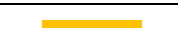 \\
\hline S11 & Mosaic in citrus & 0.95 & 0.92 & 0.92 & 0.98 & \\
\hline S12 & Scab in citrus & 0.93 & 0.92 & 0.92 & 0.95 & \\
\hline S13 & Sooty mold in citrus & 0.94 & 0.92 & 0.93 & 0.99 & \\
\hline S14 & Variegated chlorosis in citrus & 0.96 & 0.96 & 0.96 & 0.99 & \\
\hline S15 & $\begin{array}{l}\text { Cylindrocladium leaf spot } \\
\text { in coconut }\end{array}$ & 0.93 & 0.91 & 0.91 & 0.99 & - \\
\hline S16 & Lixa grande in coconut & 0.94 & 0.92 & 0.91 & 0.99 & - \\
\hline S17 & Lixa pequena in coconut & 0.91 & 0.89 & 0.89 & 0.98 & 2 \\
\hline S18 & Bacterial blight in coffee & 0.93 & 0.9 & 0.89 & 0.99 & \\
\hline S19 & Blister spot in coffee & 1 & 0.9 & 0.92 & 0.99 & \\
\hline S20 & Rust in coffee & 0.91 & 0.92 & 0.91 & 0.99 & \\
\hline $\mathrm{S} 21$ & $\begin{array}{l}\text { Northern corn leaf blight } \\
\text { in corn }\end{array}$ & 0.9 & 0.88 & 0.88 & 0.97 & \\
\hline $\mathrm{S} 22$ & Phaeosphaeria leaf spot in corn & 0.95 & 0.93 & 0.92 & 0.99 & \\
\hline $\mathrm{S} 23$ & Physoderma brown spot in corn & 0.94 & 0.93 & 0.92 & 0.98 & \\
\hline $\mathrm{S} 24$ & Southern corn rust in corn & 0.89 & 0.89 & 0.88 & 0.98 & \\
\hline $\mathrm{S} 25$ & Southern leaf blight in corn & 0.89 & 0.89 & 0.89 & 0.98 & \\
\hline S26 & Tropical rust in corn & 0.92 & 0.9 & 0.9 & 0.99 & \\
\hline $\mathrm{S} 27$ & Areolate mildew in cotton & 0.94 & 0.92 & 0.93 & 0.99 & \\
\hline $\mathrm{S} 28$ & $\begin{array}{l}\text { Myrothesium leaf spot } \\
\text { in cotton }\end{array}$ & 0.92 & 0.94 & 0.92 & 0.99 & \\
\hline S29 & Anthracnose in dry bean & 0.91 & 0.91 & 0.9 & 0.99 & \\
\hline $\mathrm{S} 30$ & Hedylepta indicata in dry bean & 0.92 & 0.92 & 0.91 & 0.99 & \\
\hline S31 & Phytotoxicity in dry bean & 0.96 & 0.96 & 0.96 & 0.99 & \\
\hline S32 & Powdery mildew in dry bean & 0.91 & 0.91 & 0.91 & 0.99 & \\
\hline S33 & Rust in dry bean & 0.89 & 0.89 & 0.88 & 0.99 & \\
\hline S34 & Target leaf spot in dry bean & 0.93 & 0.93 & 0.91 & 0.99 & \\
\hline S35 & Bacterial canker in grapevine & 0.89 & 0.88 & 0.88 & 0.98 & \\
\hline S36 & Downy mildew in grapevine & 0.93 & 0.89 & 0.9 & 0.99 & \\
\hline S37 & Powdery mildew in grapevine & 0.91 & 0.92 & 0.91 & 0.95 & \\
\hline S38 & Rust in grapevine & 0.91 & 0.9 & 0.9 & 0.99 & \\
\hline S39 & Bacterial spot in passion fruit & 0.93 & 0.91 & 0.93 & 0.99 & \\
\hline $\mathrm{S} 40$ & Blast in rice & 0.92 & 0.91 & 0.91 & 0.99 & 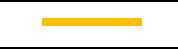 \\
\hline S41 & Bacterial blight in soybean & 0.95 & 0.93 & 0.92 & 0.98 & \\
\hline $\mathrm{S} 42$ & Brown spot in soybean & 0.92 & 0.92 & 0.92 & 0.97 & \\
\hline S43 & Downy mildew in soybean & 0.96 & 0.98 & 0.97 & 0.99 & \\
\hline $\mathrm{S} 44$ & Mosaic virus in soybean & 0.98 & 0.98 & 0.97 & 0.99 & \\
\hline $\mathrm{S} 45$ & Phytotoxicity in soybean & 0.96 & 0.97 & 0.94 & 0.99 & \\
\hline S46 & Powdery mildew in soybean & 0.95 & 1 & 0.94 & 0.99 & $=$ \\
\hline S47 & Rust in soybean & 0.91 & 0.9 & 0.9 & 0.99 & 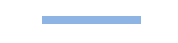 \\
\hline $\mathrm{S} 48$ & Red stripe in sugarcane & 0.93 & 0.91 & 0.91 & 0.99 & \\
\hline S49 & Ring spot in sugarcane & 0.91 & 0.88 & 0.89 & 0.99 & 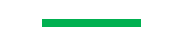 \\
\hline $\mathrm{S} 50$ & Rust in sugarcane & 0.91 & 0.91 & 0.91 & 0.99 & \\
\hline S51 & Powdery mildew in wheat & 0.92 & 0.91 & 0.9 & 0.99 & 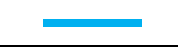 \\
\hline S52 & Rust in wheat & 0.92 & 0.89 & 0.91 & 0.99 & - \\
\hline- & Average (weighted) & 0.95 & 0.94 & 0.94 & - & - \\
\hline
\end{tabular}

\footnotetext{
* The ROC_AUC column in the table is with reference to Fig. 6.
} 


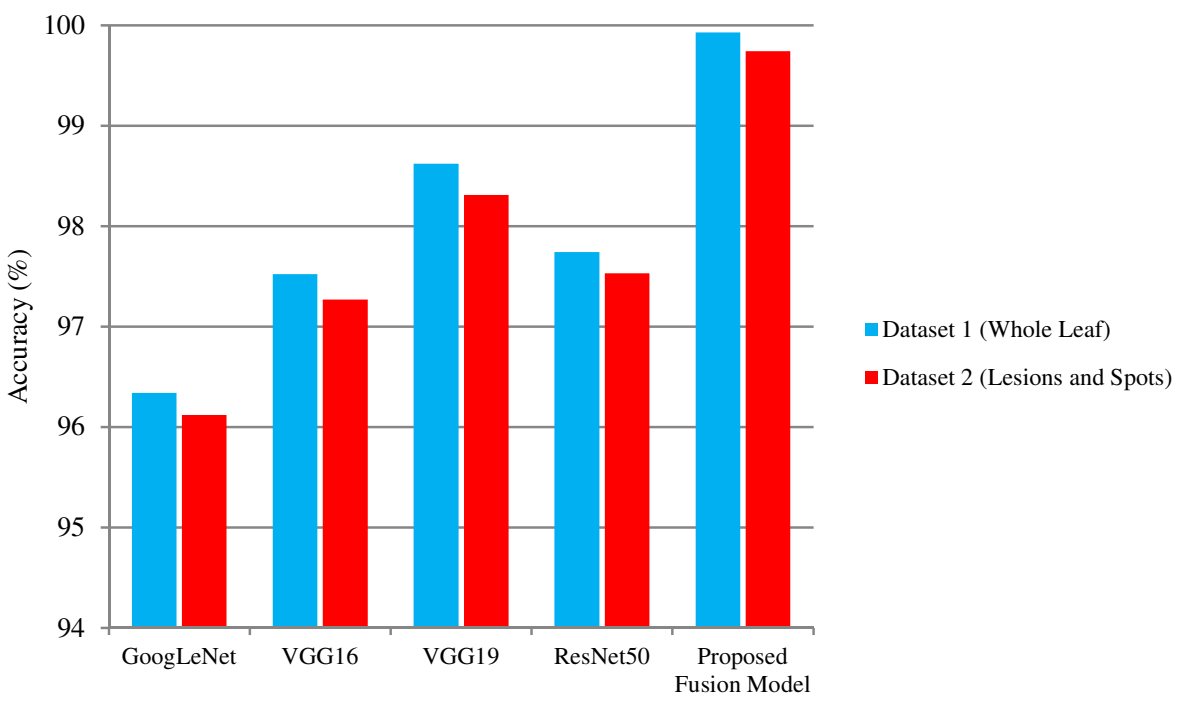

Fig. 7 Comparison of the proposed fusion model with the state-of-art methods

\section{Conclusions}

The study presented an efficient utilization of deep learning potential with traditional machine learning methods by combining deep features with HCF. A framework for automatic crop disease detection using feature fusion is proposed in the study. All the experiments were carried out by using two datasets, one with the whole leaf images and the other with the image patches of lesions and spots, to check the efficacy of the model in handling the global as well as local structure.

In the first part of the experimentation, the utility of cepstral coefficients is compared with the popular HCF using conventional machine learning algorithms. Results indicated that the cepstral coefficients gave the best results compared to other conventional features. In the next phase of the experimentation, CNN architecture was used and its performance was checked with other models suggested in literature. The CNN model used in this study outperformed the suggested models in literature and traditional machine learning algorithms. The last phase involved the fusion of handcrafted cepstral coefficients with deep features. It was seen that the proposed hybrid model gave the improved results when compared to the performance of only deep features. The hybrid model was also checked with the other CNN architectures, and it was seen that the suggested fusion framework achieved better results than the existing architectures.

The results obtained from the first and last phase suggested the use of cepstral coefficients as a relevant feature for crop disease detection. The results also showed the efficient utilization of the hybrid model for crop disease detection for the whole leaf images as well as for the image patches of lesions and spots, thus contributing to the control of crop diseases to limit agricultural and economic loss. The future work will aim to test complex background images with real field conditions to make the model robust for all image types.

\section{Conflicts of Interest}

The authors declare no conflict of interest.

\section{References}

[1] A. Kamilaris and F. X. Prenafeta-Boldú, "Deep Learning in Agriculture: A Survey," Computers and Electronics in Agriculture, vol. 147, pp. 70-90, April 2018.

[2] R. Su, T. Liu, C. Sun, Q. Jin, R. Jennane, and L. Wei, "Fusing Convolutional Neural Network Features with Hand-Crafted Features for Osteoporosis Diagnoses," Neurocomputing, vol. 385, pp. 300-309, April 2020. 
[3] C. S. Vorugunti, V. Pulabaigari, R. K. S. S. Gorthi, and P. Mukherjee, "OSVFuseNet: Online Signature Verification by Feature Fusion and Depth-Wise Separable Convolution Based Deep Learning," Neurocomputing, vol. 409, pp.157-172, October 2020.

[4] A. C. Cruz, A. Luvisi, L. De Bellis, and Y. Ampatzidis, "X-FIDO: An Effective Application for Detecting Olive Quick Decline Syndrome with Deep Learning and Data Fusion,” Frontiers in Plant Science, vol. 8, 1741, October 2017.

[5] İ. Çuğu, E. Şener, Ç. Erciyes, B. Balcı, E. Akın, I. Önal, et al., "Treelogy: A Novel Tree Classifier Utilizing Deep and Hand-Crafted Representations,” https://arxiv.org/pdf/1701.08291.pdf, January 28, 2017.

[6] S. Zhang, S. Zhang, C. Zhang, X. Wang, and Y. Shi, "Cucumber Leaf Disease Identification with Global Pooling Dilated Convolutional Neural Network," Computers and Electronics in Agriculture, vol. 162, pp. 422-430, July 2019.

[7] P. Jiang, Y. Chen, B. Liu, D. He, and C. Liang, "Real-Time Detection of Apple Leaf Diseases Using Deep Learning Approach Based on Improved Convolutional Neural Networks,” IEEE Access, vol. 7, pp. 59069-59080, May 2019.

[8] H. Genshang, W. Haoyu, Z. Yan, and W. Mingzhu, "A Low Shot Learning Method for Tea Leaf's Disease Identification," Computers and Electronics in Agriculture, vol. 163, 104852, August 2019.

[9] M. Hu, X. Bu, X. Sun, Z. Yu, and Y. Zheng, "Rape Plant Disease Recognition Method of Multi-Feature Fusion Based on D-S Evidence Theory," Mathematical and Computational Applications, vol. 22, no. 1, 18, March 2017.

[10] S. Bansal and A. Kumar, "A Post-Processing Fusion Framework for Deep Learning Models for Crop Disease Detection," IOP Conference Series: Materials Science and Engineering, vol. 998, no. 1, 012065, August 2020.

[11] J. Liu and X. Wang, "Tomato Diseases and Pests Detection Based on Improved Yolo V3 Convolutional Neural Network," Frontiers in Plant Science, vol. 11, 898, June 2020.

[12] S. Cakır, "Cepstral Methods for Image Feature Extraction," Ph.D. dissertation, Department of Electrical and Electronics Engineering and the Institute of Engineering and Science, Bilkent University, 2010.

[13] S. Gupta, J. Jaafar, W. W. Ahmad, and A. Bansal, "Feature Extraction Using MFCC," Signal \& Image Processing: An International Journal, vol. 4, no. 4, pp. 101-108, August 2013.

[14] F. G. Hashad, T. M. Halim, S. M. Diab, B. M. Sallam, and F. E. Abd El-Samie, "Fingerprint Recognition Using Mel-Frequency Cepstral Coefficients," Pattern Recognition and Image Analysis, vol. 20, no. 3, pp. 360-369, September 2010.

[15] S. S. Barpanda, B. Majhi, P. K. Sa, A. K. Sangaiah, and S. Bakshi, "Iris Feature Extraction through Wavelet Mel-Frequency Cepstrum Coefficients," Optics \& Laser Technology, vol. 110, pp. 13-23, February 2019.

[16] M. Awad, F. G. Hashad, M. M. A. Elnaby, S. E. E. Khamy, O. S. Faragallah, A. M. Abbas, et al., "Resolution Enhancement of Images for Further Pattern Recognition Applications," Optik, vol. 127, no. 1, pp. 484-492, January 2016.

[17] R. Bhagwat and Y. Dandawate, "Comprehensive Multilayer Convolutional Neural Network for Plant Disease Detection," International Journal of Advanced Computer Science and Applications, vol. 12, no. 1, pp. 204-211, 2021.

[18] D. Hughes and M. Salathé, "An Open Access Repository of Images on Plant Health to Enable the Development of Mobile Disease Diagnostics,” https://arxiv.org/ftp/arxiv/papers/1511/1511.08060.pdf, April 11, 2016.

[19] J. G. A. Barbedo, L. V. Koenigkan, and T. T. Santos, “ Identifying Multiple Plant Diseases Using Digital Image Processing,” Biosystems Engineering, vol. 147, pp. 104-116, July 2016.

[20] J. G. A. Barbedo, L. V. Koenigkan, B. A. Halfeld-Vieira, R. V. Costa, K. L. Nechet, C. V. Godoy, et al., “Annotated Plant Pathology Databases for Image-Based Detection and Recognition of Diseases," IEEE Latin America Transactions, vol. 16, no. 6, pp. 1749-1757, June 2018.

[21] J. G. A. Barbedo, "Plant Disease Identification from Individual Lesions and Spots Using Deep Learning," Biosystems Engineering, vol. 180, pp. 96-107, April 2019.

[22] M. Sharif, M. A. Khan, Z. Iqbal, M. F. Azam, M. I. U. Lali, and M. Y. Javed, "Detection and Classification of Citrus Diseases in Agriculture Based on Optimized Weighted Segmentation and Feature Selection," Computers and Electronics in Agriculture, vol. 150, pp. 220-234, July 2018.

[23] V. Singh and A. K. Misra, "Detection of Plant Leaf Diseases Using Image Segmentation and Soft Computing Techniques," Information Processing in Agriculture, vol. 4, no. 1, pp. 41-49, March 2017.

[24] A. E. Hassanien, T. Gaber, U. Mokhtar, and H. Hefny, "An Improved Moth Flame Optimization Algorithm Based on Rough Sets for Tomato Diseases Detection,” Computers and Electronics in Agriculture, vol. 136, pp. 86-96, April 2017.

[25] X. E. Pantazi, D. Moshou, and A. A. Tamouridou, “Automated Leaf Disease Detection in Different Crop Species through Image Features Analysis and One Class Classifiers," Computers and Electronics in Agriculture, vol. 156, pp. 96-104, January 2019. 
[26] S. S. Chouhan, A. Kaul, U. P. Singh, and S. Jain, "Bacterial Foraging Optimization Based Radial Basis Function Neural Network (BRBFNN) for Identification and Classification of Plant Leaf Diseases: An Automatic Approach towards Plant Pathology," IEEE Access, vol. 6, pp. 8852-8863, February 2018.

[27] C. S. Hlaing and S. M. M. Zaw, “Tomato Plant Diseases Classification Using Statistical Texture Feature and Color Feature," IEEE/ACIS 17th International Conference on Computer and Information Science, June 2018, pp. 439-444.

[28] G. Geetharamani and A. Pandian, "Identification of Plant Leaf Diseases Using a Nine-Layer Deep Convolutional Neural Network," Computers \& Electrical Engineering, vol. 76, pp. 323-338, June 2019.

[29] S. Coulibaly, B. Kamsu-Foguem, D. Kamissoko, and D. Traore, "Deep Neural Networks with Transfer Learning in Millet Crop Images," Computers in Industry, vol. 108, pp. 115-120, June 2019.

[30] M. G. Selvaraj, A. Vergara, H. Ruiz, N. Safari, S. Elayabalan, W. Ocimati, et al., "AI-Powered Banana Diseases and Pest Detection," Plant Methods, vol. 15, no. 1, pp. 1-11, August 2019.

Copyright $(\mathrm{C}$ by the authors. Licensee TAETI, Taiwan. This article is an open access article distributed under the terms and conditions of the Creative Commons Attribution (CC BY-NC) license (https://creativecommons.org/licenses/by-nc/4.0/). 